\title{
Public health policies and management strategies for genital Chlamydia trachomatis infection
}

\author{
This article was published in the following Dove Press journal: \\ Risk Management and Healthcare Policy \\ I8 May 201I \\ Number of times this article has been viewed
}

\author{
Kelly Shaw ${ }^{1,2}$ \\ David Coleman' \\ Maree O'Sullivan' \\ Nicola Stephens ${ }^{3}$ \\ 'Department of Health and Human \\ Services, Hobart, Tasmania, Australia; \\ ${ }^{2} \mathrm{Health}$ and Wellbeing Research \\ Cluster, Southern Cross University, \\ New South Wales, Australia; ${ }^{3} \mathrm{NSW}$ \\ Health, Sydney, New South Wales, \\ Australia
}

\begin{abstract}
Genital Chlamydia trachomatis is a sexually transmissible bacterial infection that is asymptomatic in the majority of infected individuals and is associated with significant short-term and long-term morbidity. The population prevalence of the infection appears to be increasing. C. trachomatis is of public health significance because of the impacts of untreated disease on reproductive outcomes, transmission of other sexually acquired infections, and the costs to health systems. At the individual level, C. trachomatis infection is readily treatable with antibiotics, although antibiotic resistance appears to be increasing. At the population level, public health control of spread of infection is more problematic. Approaches to control include primary preventive activities, increased access to testing and treatment for people with or at risk of infection, partner notification and treatment, and screening either opportunistically or as part of an organized population screening program. A combination of all of the above approaches is likely to be required to have a significant effect on the burden of disease associated with genital chlamdyia infection and to reduce population prevalence. The development of a vaccine for genital chlamydia infection could significantly reduce the public health burden associated with infection; however a vaccine is not expected to be available in the near future.
\end{abstract}

Keywords: chlamydia, public health, health policy, screening

\section{Introduction}

Chlamydia trachomatis is a gram-negative bacterium whose sexually transmissible strains D to K cause largely asymptomatic genital tract infections in males and females. ${ }^{1}$ These strains of $C$. trachomatis can also cause sexually transmissible rectal and, rarely, pharyngeal infections. Strains 1.1, 1.2, and 1.3 cause lymphogranuloma venereum, a sexually transmissible infection responsible for outbreaks of ulcerative proctitis which may be associated with inguinal and pelvic abscesses and anal strictures; the infection more commonly affects men who have sex with men. ${ }^{2}$

Risk factors for contracting $C$. trachomatis infection include having multiple sexual partners, having a new sexual partner, inconsistently using barrier contraceptives, having a history of previous or coexistent sexually transmissible infections, age (those aged 15 to 24 years most affected) and race. ${ }^{1,3}$ The likelihood of transmission of $C$. trachomatis per act of unprotected intercourse is between $30 \%$ and $50 \%$; in heterosexual couples the likelihood of a long-term sexual partner becoming infected is approximately $66 \% .^{4-6}$

In women, $C$. trachomatis commonly results in cervicitis and urethritis; up to $40 \%$ of untreated cases progress to pelvic inflammatory disease. ${ }^{7}$ Of those with pelvic inflammatory disease, up to $20 \%$ become infertile, $18 \%$ develop chronic pelvic pain,
Correspondence: Kelly Shaw

Health and Wellbeing Research Cluster Southern Cross University, Hogbin Drive, Coffs Harbour 2450, NSW, Australia

Tel +6I 266593626

Email kelly.shaw@scu.edu.au 
and $9 \%$ may have a tubal pregnancy. ${ }^{8,9}$ Chlamydia infection may also increase the risk for cervical cancer. ${ }^{10}$

In men, chlamydia infection can cause nongonococcal urethritis, acute epididymitis and, rarely, may result in urethral strictures and/or Reiters Syndrome. In males, chlamydia urethritis may also be associated with male infertility. ${ }^{8,11,12}$

C. trachomatis may be associated with adverse pregnancy outcomes for both mother and child, including miscarriage, preterm labor, premature rupture of the membranes, and low birthweight. ${ }^{13}$ Chlamydia cervicitis may be associated with neonatal conjunctivitis, nasopharyngitis, and pneumonia.

A major concern with chlamydia infections is that $70 \%$ to $85 \%$ of infected women and over $50 \%$ of infected men are asymptomatic. ${ }^{14,15}$

However, undiagnosed infection does not always result in serious complications. The natural history of untreated lower genital tract C. trachomatis infection in women is one of spontaneous clearance within 2 to 3 years of between $30 \%$ and $50 \% .{ }^{16}$ In order for tubal scarring to occur, chronic persistent infection or frequent re-infection appears to be necessary. ${ }^{12}$

\section{Epidemiology}

Although C. trachomatis is considered the most prevalent sexually transmissible infection worldwide, its true prevalence is unknown. The World Health Organization estimates that each year almost 100 million new cases occur worldwide. ${ }^{17}$

The prevalence of $C$. trachomatis reported in countries internationally varies widely among populations. Age is the strongest predictor of risk. A systematic review of C. trachomatis among asymptomatic European women estimated the prevalence to be up to $17 \%$ in some population groups and is particularly elevated among young women attending sexual health clinics. ${ }^{18}$ In population cohorts under 30 years of age in Denmark, the Netherlands, and the UK the prevalence has been estimated at between $2 \%$ and $6 \% .{ }^{19-21}$

Most epidemiological studies have been conducted in developed countries. As a result, the prevalence of the infection in populations in less developed countries is poorly understood. ${ }^{22}$

Some research suggests that the prevalence of genital C. trachomatis infections is increasing over time. However, increasing prevalence is difficult to establish definitively due to the need to control for testing effort and the effects of new testing methods in analysing rates of infection. ${ }^{23}$

The incidence rate of new infections of genital C. trachomatis is even more difficult to determine. Few studies have examined the incidence of the infection as, in order to determine the incidence of the infection, large population cohorts need to be tested at regular intervals. ${ }^{24}$

\section{Public health significance}

Chlamydia is a significant public health problem because of the impact of untreated genital chlamydia infection on rates of pelvic inflammatory disease, subfertility and poor reproductive outcomes, the association between the infection and the transmission of other sexually transmissible infections, and the impacts of the burden of disease associated with genital chlamydia infection on health services costs. Although inexpensive and effective treatment is available, control of chlamydia is challenging since most people with genital chlamydia infection are asymptomatic.

Chlamydia is costing health care systems billions of dollars to treat not only the acute infections, but also the complications they cause. ${ }^{25}$ In particular, the costs of treating subfertility due to chlamydia are high as tubal surgery and in vitro fertilization are expensive. The cost of treating the complications of undiagnosed $C$. trachomatis infection, including pelvic inflammatory disease and tubal infertility, are high both in psychosocial and in financial terms. ${ }^{24}$

As with other inflammatory sexually transmissible infections, chlamydia infection facilitates the transmission of HIV infection in both males and females. ${ }^{26}$

\section{Management strategies Clinical treatment of the infected individual}

Once diagnosed, C. trachomatis is easily and effectively treated with antibiotics. The current recommended treatments for genital tract infections caused by $C$. trachomatis are azithromycin or doxycycline. Azithromycin is preferred because of it being a single 1-g dose compared with a 7-day course of doxycycline, thereby enhancing compliance. ${ }^{27}$ Ofloxacin, minocycline, lymecycline, and erythromycin are alternative treatments, but are generally not used as regimens extend over 7 days, some are less well tolerated, and the likelihood of noncompletion of treatment is higher. ${ }^{28}$

Without antibiotics, clearance of genital chlamydia infection is poor; studies on the natural course of untreated C. trachomatis lower genital tract infections in women show spontaneous clearance rates of $30 \%$ to $50 \%$ in the first 2 to 3 years. ${ }^{29,30}$

There is emerging evidence that C. trachomatis is developing antibiotic resistance, with some clinical isolates having single and multidrug resistance when cultured in vitro. ${ }^{31-33}$ 
Further, while a shortened duration of infection from early antibiotic treatment reduces infection-associated reproductive sequelae, the number of cases continues to increase. ${ }^{34}$ Researchers have suggested that this increase is occurring because early intervention with antibiotics interferes with the development of protective immune responses, thereby increasing the risk of reinfection, which has been termed the arrested immunity hypothesis. ${ }^{34}$

The effects of this phenomenon on public health approaches to control of genital chlamydia infection are yet to be explored. Further, there is widespread use of nucleic acid amplification testing (NAAT) to detect infection. If resistance is developing, monitoring the development of this requires laboratory testing in addition to NAAT.

Testing people with genital chlamydia infection for the presence of other sexually transmissible infections is important. Coinfection with other sexually transmissible infections occurs with variable frequency among people with genital chlamydia infection. In some countries, a high prevalence of coinfection of chlamydia and gonorrhoea has been observed. Risk appears to be increased among people with genital chlamdyia who have higher numbers of sexual partners, are aged between 15 and 19 years, are in juvenile detention centers, are attending sexual health clinics, and among men who have unprotected sex with other men. ${ }^{35,36}$

\section{Public health approaches to control}

The spread of a sexually transmissible infection within a population is dependent on the probability of transmission of the pathogen from an infected to a susceptible individual, the rate of contact between infected and susceptible individuals, and how long the infection persists. ${ }^{7}$ Controlling chlamydia therefore requires interventions to reduce each of these factors.

Some factors are more easily influenced than others. The probability of transmission can be reduced by the use of condoms and the contact rate can be reduced by having fewer sexual partners and concurrent partnerships. ${ }^{7}$ A particular public health goal for chlamydia control is reducing the duration of infection; as most infected individuals are asymptomatic they are less likely to present to clinical services for diagnosis and treatment. The infected person can therefore remain infectious for a long period and can continue to transmit the infection to their sexual partners until the infection spontaneously resolves.

The range of activities required for control of genital chlamdyia infection includes:
- $\quad$ primary prevention involving people at risk of acquiring and transmitting the infection; primary preventive activities include sexual health and relationships education;

- the promotion of safer sex and condom use;

- effective diagnosis and treatment of those with infection; and

- identifying and treating partners of infected individuals. Public health initiatives aimed at influencing social and environmental determinants of health are an important component of successful community-based health promotion. By necessity, policies aimed at reducing the burden of disease associated with chlamydia infection need to include access to, and quality of, healthcare services equipped to identify and manage people with or at risk of infection. This includes ensuring access to health services for minors. ${ }^{37}$

A diversity of services is required to provide comprehensive access to patients with, or at risk of, infection. General practitioners are a mainstay of provision of assessment and management. Sexual health services, family planning, obstetrics and gynecology services, and general hospitals also play an important role in providing assessment and management. ${ }^{38}$

\section{Primary prevention}

Chlamydia is a risk for all young people who are sexually active. As a result, population-wide information and education forms the basis of primary preventive activities for chlamydia control. ${ }^{39}$ Countries with pragmatic school-based sexual health education policies have been shown to have better sexual health-related statistics among school-aged people than countries that do not. ${ }^{40}$

Primary preventive activities need to be well designed, long-term, and research-based in order to be effective. ${ }^{41}$ There is some evidence that the most successful sexual health promotion strategies are those that acknowledge the social and media influences on young people and use these to help strengthen group norms around safe sexual behavior. $^{42}$

Primary prevention of chlamydia infection is a frequent health and social policy initiative of governments internationally. The World Health Organization recommends that countries implement evidence-based strategies for primary prevention and surveillance of sexually transmissible infections. ${ }^{22}$ Governments have invested in campaigns for safer sex to increase awareness of risk behaviors. ${ }^{43}$

\section{Partner notification and treatment}

Partner notification and treatment is a common strategy for reducing transmission of genital chlamydia infection as 
the highest prevalence of chlamydia infection occurs in the partners of patients with diagnosed chlamydia infection. ${ }^{28}$ However, partner notification reaches less than $60 \%$ of partners. $^{44}$

Randomized controlled trials assessing the impact of partner notification and treatment for chlamydia have shown variable impacts on the recurrence or persistence of chlamydia infection in index patients. Some studies have found statistically significant reductions; others have found a trend towards decreased infection but this did not reach statistical significance. ${ }^{45}$

Published guidelines internationally recommend referral of sexual partners for screening and possible treatment. Recommendations regarding the time period for identifying previous partners differs slightly; the British Association for Sexual Health and HIV provides recommendations according to whether the index patient is symptomatic or asymptomatic, recommending a 4-week look-back period for symptomatic index cases, and an arbitrary cut-off of 6 months for asymptomatic index patients (or until the most recent sexual partner). ${ }^{46}$ The Scottish Intercollegiate Guidelines Network (SIGN) makes similar recommendations, but takes index patient gender into account, recommending a 4-week look-back period for men with symptomatic infection and a cut-off of 6 months for women and asymptomatic men (or until the most recent sexual partner). ${ }^{47}$ Australasian guidelines recommend contact tracing for 6 months before identification of chlamydia infection. ${ }^{4}$

The US Centers for Disease Control and Prevention (CDC) recommends that sex partners be evaluated, tested, and treated if they had sexual contact with an infected patient during the 60 days before onset of symptoms or diagnosis. However, they also recommend evaluation and treatment of the last sexual contact, even if that contact was more than 60 days before symptom onset. ${ }^{48}$

CDC and SIGN address patient delivered partner medication (PDPM). The groups agree that some evidence demonstrates that it can reduce the risk of persistent or recurrent infection in patients with chlamydia compared with standard partner referral. SIGN notes, however, that PDPM cannot currently be carried out in the UK because of legal considerations. These include the uncertain legal status of the practice of medical and pharmacy boards in some countries, uncertainties regarding the duty of care of treating clinicians to assess patients before dispensing medications with the potential for adverse effects, and the lack of investigation of risk factors for coinfection. ${ }^{49,50} \mathrm{CDC}$ discusses PDPM for use only in heterosexual sexual partnerships.
They add that patient-delivered partner therapy is not routinely recommended for men who have sex with men because of a high risk for coexisting infections, especially undiagnosed HIV infection, in their partners.

\section{Presumptive treatment}

Periodic treatment given for a presumed infection, in the absence of investigations to confirm presence of infection, has been proposed as a strategy to reduce the prevalence of infections in populations who are at risk. However, results of studies examining this approach have failed to establish its effectiveness. Randomized trials of monthly antibiotics provided to sex workers did not uniformly demonstrate a reduction in the incidence of chlamydia infections. ${ }^{51} \mathrm{~A}$ randomized trial comparing prophylaxis with a screen and treat strategy for chlamydia management in patients presenting for termination of pregnancy demonstrated that prophylaxis was as effective and more cost effective. ${ }^{52}$ Mathematical modeling to estimate the impact of periodic presumptive treatment on the transmission of genital chlamydia infection by female sex workers has shown significant decreases in the prevalence of genital chlamydia with $>30 \%$ coverage of the sex worker population. ${ }^{53}$

Disadvantages of prophylactic treatment are that partner notification and treatment does not occur, reducing the opportunity to limit population spread of the infection.

\section{Self-testing with point-of-care tests}

Point-of-care tests provide people with a rapid test that can be used to self-test for the presence of chlamydia infection. These tests have a reported sensitivity of $52 \%$ to $80 \%$, specificity of $97 \%$ to $99 \%$; lower than reported sensitivity and specificity of NAAT available through laboratories. ${ }^{28}$

There is currently insufficient evidence to demonstrate that using point-of-care-tests would increase the overall number of cases of chlamydia detected. As the sensitivity and specificity of these tests is lower than tests used in current practice, increased number of people incorrectly diagnosed, and number of contacts incorrectly traced and managed result from their use; for a hypothetical cohort of 1000 people, using the current practice of NAAT would result in 13 people who were offered testing being correctly treated whereas the point-of-care test would result in between 7 and 11 people being correctly treated. ${ }^{28}$

\section{Screening for genital chlamydial infection}

In order to reduce the impacts of $C$. trachomatis infection, screening programs have been introduced in a number of 
countries worldwide. The primary aim of screening is to reduce morbidity by early detection and treatment of uncomplicated lower genital tract infection. A secondary aim of screening is to reduce population prevalence of the infection. ${ }^{54}$

Screening can be population-based and systematic or can be opportunistic. In opportunistic screening, patients are offered screening whilst attending their treating health professional. In most countries where chlamydia screening guidelines exist, opportunistic screening of patients at risk of acquiring the infection is promoted. ${ }^{54,55}$

Different screening methods are adapted to different healthcare settings and different screening approaches are generally selected to suit the particular healthcare setting. For example, outpatient clinics accessed by at-risk youth and/or men who have sex with men may routinely screen all patients accessing the clinic; in contrast, screening in a general practice setting is more appropriately directed towards testing of patients in whom risk factors for genital chlamydia infection are present. ${ }^{38}$

A direct comparison of the recommendations presented in UK and US guidelines for the management of $C$. trachomatis infection was conducted by the US National Guideline Clearinghouse ${ }^{55}$ According to current guidelines:

- The CDC and US Preventive Services Task Force (USPSTF) recommend routine screening of all nonpregnant sexually active women aged 25 years and 24 years or younger respectively, as well as older nonpregnant women at increased risk (eg, those who have a new sex partner or multiple sex partners). With regard to frequency of screening, CDC recommends annual screening in these populations. USPSTF cites the CDC recommendation, but states that the optimal interval for screening for nonpregnant women is unknown; ${ }^{56,57}$

- In contrast with CDC and USPSTF guidelines, SIGN recommends that testing be targeted at those individuals identified as belonging to groups with the highest prevalence of infection: sexual partners of chlamydia-positive individuals, sexual partners of those with suspected but undiagnosed chlamydial infection, those who have been diagnosed with chlamydia in the previous 12 months, all patients (including men who have sex with men) attending genitor-urinary medicine clinics, and women undergoing termination of pregnancy. While SIGN does not recommend routine screening in sexually active women aged 24 years or younger, they do recommend that resources for chlamydia testing in women be targeted where prevalence is known to be highest, that is, first at those aged 15 to 19 and then at those aged 20 to 24;
- With regard to screening of pregnant women, USPSTF recommends screening for all pregnant women aged 24 and younger, and for older pregnant women who are at increased risk. CDC states that prenatal screening of pregnant women can prevent chlamydial infection among neonates, and that pregnant women aged $<25$ years are at high risk for infection. They add that local or regional prevalence surveys of chlamydial infection can be conducted to confirm the utility of using these recommendations in particular settings. According to SIGN, there is no evidence to suggest that pregnancy alone should be an indication for routine testing for chlamydia;

- None of the groups recommends routine screening of sexually active men. USPSTF concluded that the current evidence is insufficient to assess the balance of benefits and harms of screening for chlamydial infection for men. CDC similarly found that evidence is insufficient to recommend routine screening in sexually active young men. However, CDC adds that screening of sexually active young men should be considered in clinical settings with a high prevalence of chlamydia (eg, adolescent clinics, correctional facilities, and STD clinics).

Australian guidelines not included in the National Clearinghouse Review recommend screening for $C$. trachomatis infection in all sexually active females under 25 years of age every 12 months. Screening of sexually active males is not supported with the exception of men who have sex with men, who should be screened every 12 months. ${ }^{58}$

As the objectives of a screening program not only target the individual level (reducing complications by early diagnosis and treatment), but also the public health level (reducing transmission within the population) sufficient program uptake is essential for screening to be effective.

Opportunistic screening results in variable rates of testing within target populations. For example, in the US, the annual screening rate in sexually active 15 - to 25 -year-olds is approximately $41 \% .^{59}$ In Sweden, $71 \%$ of women have had at least one chlamydia test in a 10 -year period. ${ }^{60}$ In the UK in $2009 / 10,22.1 \%$ of 15 - to 24-year-olds have been tested for genital chlamydia. ${ }^{61}$ In contrast, in Australia less than $10 \%$ of people in the target screening groups have had a chlamydia test. ${ }^{62}$

In comparison, a trial of population screening in the Netherlands resulted in between $37 \%$ and $41 \%$ participation. ${ }^{21}$

The public health impacts of screening, both opportunistic and population-based, are poorly understood and few studies have been performed to demonstrate public health effects. A US randomized controlled trial of population 
screening demonstrated a $56 \%$ reduction in pelvic inflammatory disease. ${ }^{63} \mathrm{~A}$ Danish randomized trial in school students and young people found a $50 \%$ reduction in pelvic inflammatory disease that resulted from population-based screening. In contrast, a UK-based opportunistic screening program conducted using self-collected specimens in females failed to demonstrate an association between chlamydia screening and pelvic inflammatory disease. ${ }^{64}$ Ecological studies in Sweden and the US have shown that chlamydia prevalence and complications have decreased after screening has been implemented. However, it is uncertain whether the screening program was responsible for the decrease that was observed.$^{65}$ Recent analysis of claims data also suggests that screening is associated with decreases in pelvic inflammatory disease. ${ }^{66}$

Even though the rates of genital tract infections continue to rise, there are no uniformly accepted screening practices. ${ }^{65}$ Further, a recent systematic review has questioned the effectiveness of opportunistic chlamydia screening, because there is little evidence in the available literature to demonstrate that the practice is effective. ${ }^{67}$ Screening appears to be costeffective when the costs related to diagnosing and treating the late sequelae of infection exceed or at least largely offset the costs related to screening people and treating positive cases. Systematic reviews of the cost effectiveness of screening young, asymptomatic women suggest that screening is cost effective, mainly because of the reduction in long-term health costs. ${ }^{68}$

\section{Surveillance}

Surveillance activities are an important public health management strategy for chlamydia prevention and control. If we are to have policies and new management strategies how do we measure/evaluate their effectiveness? Surveillance is one tool.

The primary role of public health surveillance is to guide the planning and evaluation of policy and programs, through the collection, analysis, and interpretation of statistical information. The main form of chlamydia surveillance is passive reporting of cases to health departments by doctors or laboratories. ${ }^{69,70}$

Passive surveillance can be established on an ongoing basis, provide geographic coverage, and can be performed using routinely collected administrative data, reducing resource implications. However, passive surveillance is biased by testing effort for infection. Also, passive surveillance data do not provide information on characteristics such as gender and risk factors for acquiring infection. ${ }^{71}$
Passive surveillance can be supplemented through enhanced surveillance, the collection of additional information on some or all notifications received through passive surveillance systems, or sentinel surveillance, the use of selected clinical sites to collect systematic data on cases of infection that are identified. Such data can be used to evaluate clinic-based initiatives, broader prevention programs, and help interpret trends in passive surveillance..$^{72,73}$

\section{Vaccine}

The development of a vaccine for genital chlamydia infection could significantly reduce the public health burden associated with infection. Results of vaccine trials have to date been disappointing. Multiple approaches to vaccine development have been trialed but immunity to the specific serovars that cause genital tract infection, which is long-lasting, is difficult to elicit. At present, it is not expected that a vaccine will be available within the next 10 years. ${ }^{1,24}$

\section{Summary}

Most people with genital chlamydia infection are asymptomatic and remain unnoticed and untreated. People with untreated infection may be at risk of complications and determine the reservoir for onward transmission in the population. Strategies for the control of infection and prevention of its complications are only partially effective ie, primary prevention and partner notification, or are not yet available, ie, a vaccine. Randomized controlled trials that assess the effectiveness of many chlamydia control strategies are inconclusive or have not been performed.

Screening is an additional strategy for early detection and treatment of infected cases. Evidence on the impact of screening on the prevalence of chlamydia infections at a population level is still limited as is the impact on the prevalence of complications in screened women.

In spite of these evidence limitations, it is likely that a range of public health approaches to control of genital C. traachomatis infection need to be employed simultaneously in order to be effective in reducing the burden of disease associated with infection and to reduce population prevalence. The four levels of public health intervention that should be incorporated into chlamydia control programs include:

1. Primary prevention: including health promotion and education, and access to condoms;

2. Diagnosis and management services: ensuring the reach and target effectiveness of clinical services for the diagnosis and management of people with infection, including those in hard-to-reach population groups at risk of 
infection, and provision of effective partner notification and management services in accordance with evidencebased guidelines;

3. Provision of opportunistic testing to population groups at risk of infection: testing may be provided via clinical services or through mechanisms that encourage selftesting; and

4. Provision of opportunistic or systematic population screening: although evidence is mixed, the organized provision of regular chlamydia testing to cover a substantial proportion of at-risk population groups is likely to be necessary to reduce chlamydia prevalence within the population.

Introducing a screening program for chlamydia needs to be considered with the same care as any other screening program, with an assessment of all the potential benefits, harms, and costs.

Effective resourcing and implementation of national chlamydia control strategies requires leadership and commitment from healthcare policy makers. The most appropriate national strategies are likely to vary across countries, and national strategies should be developed in consultation with professional medical organizations, and funders and providers of healthcare and diagnostic services. Surveillance activities are an essential component of holistic public health responses to chlamydia infection, to provide information regarding the success of preventive and management activities at the population level.

Further, interventions targeting the social determinants of health and their impact on chlamydia prevalence and outcomes have not been the subject of extensive research. The sociodemographic characteristics of population subgroups in whom genital chlamydia infection is most prevalent suggest that holistic public health solutions targeting social determinants should also form the basis of public health policies for chlamydia prevention and control.

\section{Disclosure}

The authors declare no conflicts of interests in this work.

\section{References}

1. Carey A, Beagley K. Chlamydia trachomatis, a hidden epidemic: effects on female reproduction and options for treatment. Am J Repro Immunol. 2010;63:576-586.

2. Blank S, Schillinger J, Harbatkin D. Lymphogranuloma venereum in the industrialised world. Lancet. 2005;365:1607-1608.

3. Nelson H, Helfand M. Screening for chlamydial infection. Am J Prev Med. 2001;20:95-107.

4. Australasian Society for HIV Medicine. Contact Tracing Manual. 2010. http://ctm.ashm.org.au/Default.asp?PublicationID=6. Accessed April 6, 2011.
5. Knauper B, Kornik R. Perceived transmissibility of STIs: lack of differentiation between HIV and Chlamydia. Sex Transm Inf. 2004; 80:74.

6. Lycke E, Lowhagen G, Hallhagen $\mathrm{G}$, et al. The risk of transmission of genital Chlamydia trachomatis infection is less than that of genital Neisseria gonorrhoeae infection. Sex Transm Dis. 1980;7:6-10.

7. Anderson R, May R. Infectious Diseases of Humans. Oxford: Oxford University Press: 1991.

8. Peipert J. Genital chlamydial infections. N Engl J Med. 2003;349: 2424-2430.

9. Silins I, Ryd W, Strand A, et al. Chlamydia trachomatis infection and persistence of human papillomavirus. Int J Cancer. 2005;116:110-115.

10. Wallin K, Wiklund F, Luostarinen T, et al. A population-based prospective study of Chlamydia trachomatis infection and cervical carcinoma. Int J Cancer. 2002;101:371-374.

11. Gonzales G, Munoz C, Suchez R, et al. Update on the impact of Chlamydia trachomatis infection on male fertility. Andrologia. 2004; 36:1-23

12. Mardh $P$. Tubal factor infertility, with special regard to chlamydial salpingitis. Curr Opin Infect Dis. 2004;17:49-52.

13. Baud D, Regan L, Greub G. Emerging role of Chlamydia and Chlamydia-like organisms in adverse pregnancy outcomes. Curr Opin Infect Dis. 2008;21:70-76.

14. Miller W, Ford C, Morris M, et al. Prevalence of chlamydial and gonococcal infections among young adults in the United States. JAMA. 2004;291:2229-2236.

15. Risser W, Bortot A, Benjamins L, et al. The epidemiology of sexually transmitted infections in adolescents. Semin Pediatr Infect Dis. 2005; 16:160-167.

16. Geisler W, Wang C, Morrison S, Black C, Bandea C, Hook E. The natural history of untreated Chlamydia trachomatis infection in the interval between screening and returning for treatment. Sex Trans Dis. 2008;35:119-123.

17. WHO. Estimated New Cases of Chlamydial Infections Among Adults, 1999. Available at: http://www.who.int/docstore/hiv/GRSTI/pdf/ figure04.pdf. Accessed April 6, 2011.

18. Wilson J, Honey E, Templeton A, et al. EU Biomed Concerted Action Group. A systematic review of the prevalence of Chlamydia trachomatis among European women. Hum Reprod Update. 2002;28:385-394.

19. Andersen B, Olesen F, Moller J, Ostergaard L. Population-based strategies for outreach screening of urogenital Chlamydia trachomatis infections: a randomized, controlled trial. J Infect Dis. 2002;185:252-258.

20. Macleod J, Salisbury C, Low N, et al. Coverage and uptake of systematic postal screening for genital Chlamydia trachomatis and prevalence of infection in the United Kingdom general population: cross sectional study. BMJ. 2005;330:940-942.

21. Van Bergen J, Gotz H, Richardus J, Hoebe C, Broer J, Coenen A. Prevalence of urogenital Chlamydia trachomatis increases significantly with level of urbanization and suggests targeted screening approaches: results from the first national population based study in the Netherlands. Sex Trans Infect. 2005;81:17-23.

22. WHO. Prevention and control of sexually transmitted infections: draft global strategy. Available at: http://www.who.int/reproductive-health/ docs/stis_strategy.pdf. Accessed April 6, 2011.

23. Fine D, Dicker L, Mosure D, Berman S. Increasing Chlamydia positivity in women screened in family planning clinics: do we know why? Sex Trans Dis. 2008;35:47-52.

24. Land J, Van Bergen J, Morre S, Postma M. Epidemiology of Chlamydia trachomatis infection in women and the cost-effectiveness of screening. Hum Reprod Update. 2009;16:189-204.

25. Beagley K, Timms P. Chlamydial trachomatis infection: incidence, health costs and prospects for vaccine development. Am J Reprod Immunol. 2000;48:47-68.

26. Baeten J, Overbaugh J. Measuring the infectiousness of persons with HIV-1: opportunities for preventing sexual HIV-1 transmission. Curr HIV Res. 2003;1;69-86.

27. Adimora Adaora A. Treatment of uncomplicated genital Chlamydia trachomatis infections in adults. Clin Infect Dis. 2002;35:S183-S186. 
28. Hislop J, Quayyum Z, Flett G, Boachie C, Fraser C, Mowatt G. Systematic review of the clinical effectiveness and cost-effectiveness of rapid point-of-care tests for the detection of genital Chlamydia infection in women and men. Health Technol Assess. 2010;14:1-97, iii-iv.

29. Golden M, Schillinger J, Markowitz L, St Louis M. Duration of untreated genital infections with Chlamydia trachomatis: a review of the literature. Sex Transm Dis. 2000;27:329-337.

30. Geisler W, Wang C, Morrison S, Black C, Bandea C, Hook E. The natural history of untreated Chlamydia trachomatis infection in the interval between screening and returning for treatment. Sex Transm Dis. 2008;35:119-123.

31. Katz B, Fortenberry D, Orr D. Factors affecting chlamydial persistence or recurrence one and three months after treatment. In: Stephens R. et al, editors. Chlamydia Infections: Proceedings of the Ninth International Symposium on Human Chlamydial Infections. Napa, CA, Berkeley, CA, Berkeley University Press; 1998:35-38.

32. Somani J, Bhullar V, Workowski K, Farshy C, Black C. Multiple drug-resistant Chlamydia trachomatis associated with clinical treatment failure. J Infect Dis. 2000;181:1421-1427.

33. Horner P. The case for further treatment studies of uncomplicated genital Chlamydia trachomatis infection. Sex Trans Infect. 2006;82: 340-343.

34. Brunham R, Rekart M. The arrested immunity hypothesis and the epidemiology of Chlamydia control. Sex Trans Dis. 2008;35:53-54.

35. Harindra V, Tobin J, Underhill G. Opportunistic chlamydia screening; should positive patients be screened for co-infections? Int J STD AIDS. 2002;13:821-825.

36. Miller W, Ford C, Morris M, et al. Prevalence of chlamydial and gonococcal infections among young adults in the united states. JAMA. 2004;291:2229-2236.

37. Ahern N, Kiehl E. Adolescent sexual health and practice - a review of the literature. Fam Comm Health. 2006;29:299-313.

38. Shaw K, Stephens N, Coleman D, O'Sullivan M. Role of the general practitioner in testing for genital Chlamydia trachomatis infection: an analysis of enhanced surveillance data. Sex Health. 2009;6: 208-212.

39. Ritchie G. Strategies to promote sexual health. Nurs Stand. 2006;20: 35-40.

40. Weaver H, Smith G, Kippax S. School-based sex education policies and indicators of sexual health among young people: a comparison of the Netherlands, France, Australia and the United States. Sex Education. 2005;5:171-188.

41. Shrier L. Sexually transmitted diseases in adolescents: biologic, cognitive, psychologic, behavioural and social issues. Adolesc Med Clin. 2004;15:215-234.

42. Mitchell A, Smith A. Safe sex messages for adolescents. Do they work? Aust Fam Phys. 2000;29:31-34.

43. Low N, Broutet N, Adu-Sarkodie Y, Barton P, Hossain M, Hawkes S. Global control of sexually transmitted infections. Lancet. 2006;368: 2001-2016.

44. Low N, McCarthy A, Roberts T, et al. Partner notification of chlamydia infection in primary care: randomised controlled trial and analysis of resource use. BMJ. 2006;332:14-19.

45. Saperstein A, Fimhaber G. Should you test or treat partners of patients with gonorrhoea, Chlamydia or trichomoniasis? J Fam Pract. 2010;59:46-48.

46. British Association of Sexual Health and HIV (BASHH). 2006 UK national guideline for the management of genital tract infection with Chlamydia trachomatis. London (UK): British Association of Sexual Health and HIV (BASHH); 2006.

47. Scottish Intercollegiate Guidelines Network (SIGN). Management of Genital Chlamydia trachomatis Infection. A National Clinical Guideline. Edinburgh (Scotland): Scottish Intercollegiate Guidelines Network (SIGN); 2009 Mar (SIGN publication; no. 109).

48. Centers for Disease Control and Prevention (CDC). Sexually transmitted diseases treatment guidelines, 2006. [Published errata appear in MMWR 2006;55:997]
49. Hocking J, Parker R, Pavlin N, Fairley C, Gunn J. What needs to change to increase chlamydia screening in general practice in Australia? the views of general practitioners. BMC Public Health. 2008;8:425.

50. Golden M, Uzoeshi A, Williams D, Handsfield H. The legal status of patient-delivered partner therapy for sexually transmitted infections in the United States. Sex Trans Dis. 2005;32:112-114.

51. Manhart L, Holmes K. Randomized controlled trials of individual-level, population-level, and multilevel interventions for preventing sexually transmitted infections: what has worked? J Infect Dis. 2005;191: S7-S24.

52. Penney G, Thomson M, Norman J, et al. A randomised comparison of strategies for reducing infective complications of induced abortion. Br J Obset Gynaecol. 1998;105:599-604.

53. Vickerman P, Ndowa F, O'Farrell N, et al. Using mathematical modelling to estimate the impact of periodic presumptive treatment on the transmission of sexually transmitted infections and HIV among female sex workers. Sex Transm Infect. 2010;86:163-168.

54. Regan D, Wilson D, Hocking J. Coverage is the key for effective screening of Chlamydia trachomatis in Australia. $J$ Infect Dis. 2008;198:349-358.

55. National Guideline Clearinghouse (NGC). Guideline synthesis: Screening for and management of chlamydial infection. In: National Guideline Clearinghouse. Rockville (MD): 2001 May (revised 2010 Feb). http://www.guideline.gov. Accessed April 6, 2011.

56. US Preventive Services Task Force (USPSTF). Screening for chlamydial infection: recommendation statement. Ann Intern Med. 2007;147: $128-134$.

57. Sexually Transmitted Diseases Treatment Guidelines, 2010. MMRW 2010;59:RR-12. Available at: http://www.cdc.gov/std/treatment/2010/ STD-Treatment-2010-RR5912.pdf. Accessed April 6, 2011.

58. Royal Australian College of General Practitioners. Guidelines for Preventive Activities in General Practice. 7th ed; 2009. http://www. racgp.org.au/Content/NavigationMenu/ClinicalResources/RACGPGuidelines/TheRedBook/redbook_7th_edition_May_2009.pdf. Accessed April 6, 2011.

59. Centers for Disease Control and Prevention. Chlamydia screening among sexually active young female enrollees of health plans, Chlamydia epidemiology and screening United States, 2000-2007. MMWR. 58:362-365. http://www.cdc.gov/mmwr/preview/mmwrhtml/ mm5814a2.htm?s_cid=mm5814a2_e. Accessed April 6, 2011.

60. Low N, Egger M, Sterne J, et al. Incidence of severe reproductive tract complications associated with diagnosed genital Chlamydia infection: the Uppsala Women's Cohort Study. Sex Trans Infect. 2006;82:212-218.

61. National Chlamydia Screening Programme. Full year data for 2009/2010. http://www.chlamydiascreening.nhs.uk/ps/media/news. html. Accessed April 18, 2011.

62. Hocking J, Fairley C, Guy R, et al. Innovative trials are still needed to investigate the effectiveness of Chlamydia screening. Rapid response. $B M J .2010$, April 14. Epub ahead of print.

63. Scholes D, Stergachis A, Heidrich F, Andrilla H, Holmes K, Stamm W. Prevention of pelvic inflammatory disease by screening for Chlamydia infection. N Engl J Med. 1996;334:1362-1367.

64. Oakeshott P, Kerry S, Aghaizu A, et al. Randomised controlled trial of screening for Chlamydia trachomatis to prevent pelvic inflammatory disease: the POPI (prevention of pelvic infection) trial. BMJ. 2010; 340:1642.

65. Low N; the Screening for Chlamydia Review in Europe (SCREen) project team. Publication report on chlamydia control activities in Europe. Eurosurveillance. 2008;13:28

66. Owusu-Edusei K, Bohm M, Chesson H, et al. Chlamydia screening and pelvic inflammatory disease insights from exploratory time-series analyses. Am J Prev Med. 2010;38:652-657.

67. Low N, Bender N, Nartey L, Redmond S, Shang A, Stephenson J. Effectiveness of Chlamydia screening: systemic review. Int J Epidemiol. 2009;38:435-448.

68. Honey E, Augood C, Templeton A, et al. Cost effectiveness of screening for Chlamydia trachomatis: a review of published studies. Sex Trans Infect. 2002;78:406-412. 
69. Department of Health and Ageing (Australia). National Notifiable Diseases Surveillance System data. http://www9.health.gov.au/cda/ source/cda-index.cfm. Accessed April 6, 2011.

70. European Center for Disease Prevention and Control. Exploring the features of Chlamydia surveillance. ECDC. February 2009.

71. National Centre in HIV Epidemiology and Clinical Research. HIV/AIDS, viral hepatitis and sexually transmissible infections in Australia. Annual Surveillance Report, 2010. Sydney, NSW: National Centre in HIV Epidemiology and Clinical Research, University of New South Wales; 2010.
72. Guy R, Kong F, Goller J, et al; ACCESS Collaboration. A new national Chlamydia Sentinel Surveillance System in Australia: evaluation of the first stage of implementation. Commun Dis Intell. 2010;34:319-328.

73. Stephens N, O'Sullivan M, Coleman D, Shaw K. Chlamydia trachomatis in Tasmania 2001-2007: rising notification trends. Aust N Z J Publich Health. 2010;34:120-125.

\section{Publish your work in this journal}

Risk Management and Healthcare Policy is an international, peerreviewed, open access journal focusing on all aspects of public health, policy, and preventative measures to promote good health and improve morbidity and mortality in the population. The journal welcomes submitted papers covering original research, basic science, clinical \& epidemio- logical studies, reviews and evaluations, guidelines, expert opinion and commentary, case reports and extended reports. The manuscript management system is completely online and includes a very quick and fair peerreview system, which is all easy to use. Visit http://www.dovepress.com/ testimonials.php to read real quotes from published authors.

Submit your manuscript here: http://www.dovepress.com/risk-management-and-healthcare-policy-journal 